\title{
Atividade física habitual de mulheres da região norte e serrana de Santa Catarina
}

\author{
Camila da Cruz Ramos de Araujoํ, Alexandrina Gomes de Oliveira², Adriana Coutinho de Azevedo Guimarães³, \\ Leonessa Boing ${ }^{4}$, Melissa de Carvalho Souza ${ }^{5}$, Zenite Machado ${ }^{6}$
}

\begin{abstract}
RESUMO
O estudo transversal objetivou comparar a atividade física habitual de 705 mulheres da região Norte e Serrana de Santa Catarina. Métodos: Utilizou-se um questionário autoaplicável: a) identificação pessoal; b) situação socioeconômica - Associação Brasileira de Empresas de Pesquisa; c) Questionário Internacional de Atividade Física. Resultados: aproximadamente $72 \%$ das mulheres são suficientemente ativas com diferença significativa na prática da caminhada $(p<0,001)$, na qual as mulheres de Lages dedicavam maior quantidade de tempo quando comparadas às mulheres de Joinville $(55 \pm 51 \mathrm{~min} / \mathrm{d})$ e $(36 \pm 31 \mathrm{~min} / \mathrm{d})$, respectivamente. Conclusão: As mulheres de ambas as regiões são ativas e atendem as recomendações da Organização Mundial da Saúde (2011) para a prática de atividade física e acumulando pelo menos 30 minutos de atividade física moderada diariamente.
\end{abstract}

Descritores: Atividade Física. Prevalência. Saúde. Mulheres

\section{Habitual physical activity of women from the northern and mountainous region of Santa Catarina}

\begin{abstract}
Objective - This study aimed to compare the physical activity of 705 women from the Northern and Mountainous region of Santa Catarina. Methods - A self-administered questionnaire was used for the study: a) personal identification; b) socioeconomic status - Brazilian Association of Population Studies; c) International Physical Activity Questionnaire. Results - Approximately $72 \%$ of women are sufficiently active with a significant difference in the practice of walking $(p<0.001)$, in which women from Lages devoted most amount of time when compared to women of Joinville $(55 \pm 51$ $\mathrm{min} / \mathrm{d})$, and $(36 \pm 31 \mathrm{~min} / \mathrm{d})$, respectively. Conclusion - Women in both regions are active and follow the recommendation of the World Health Organization (2011) for physical activity and accumulate at least 30 minutes of moderate physical activity daily.
\end{abstract}

Descriptors: Motor Activity. Prevalence. Health. Women.

${ }^{1}$ Mestranda em Ciências do Movimento Humano pela Universidade do Estado de Santa Catarina (UDESC), Florianópolos, SC, Brasil ${ }^{2}$ Bacharel em Educação física pela Universidade do Estado de Santa Catarina (UDESC), Florianópolos, SC, Brasil

${ }^{3}$ Professora adjunta do Departamento de Educação Física e do Programa de Pós-graduação em Ciências do Movimento Humano no Centro de Ciências da Saúde e do Esporte da Universidade do Estado de Santa Catarina (UDESC), Florianópolis, SC, Brasil

${ }^{4}$ Mestranda em Ciências do Movimento Humano pela Universidade do Estado de Santa Catarina (UDESC), Florianópolos, SC, Brasil

${ }^{5}$ Mestranda em Ciências do Movimento Humano pela Universidade do Estado de Santa Catarina (UDESC), Florianópolos, SC, Brasil

${ }^{6}$ Doutora em Ciências da Motricidade Humana pela Universidade Técnica da Lisboa, UTL, Portugal 


\section{Introdução}

A prática de atividade física mostrou ser fator determinante para um melhor estilo de vida em estudos com populações diversificadas, nomeadamente, crianças, mulheres e idosos ${ }^{1-3}$. Sua regularidade é definida como a prática de pelos menos 30 minutos de atividade física em cinco ou mais dias na semana podendo proporcionar uma vida mais saudável, conservando o metabolismo no ritmo adequado, reduzindo os riscos de hipertensão, diabetes e vários tipos de câncer, além de possibilitar incremento das funções cardiovasculares, metabólicas, musculoesqueléticas e auxiliar no controle e redução da adiposidade corporal ${ }^{4,5}$. Outros benefícios sugerem a redução do estresse e dos sintomas da depressão e 0 aumento da sensação de bem-estar, envolvendo maiores níveis de autoconfiança e satisfação pessoal ${ }^{6}$.

Para as mulheres, a atual difusão eclodida pela modernidade e independência, proporcionou novos hábitos, rotinas profissionais e novos padrões de comportamento alterando assim as condições e qualidade de vida dessa população, resultando em mudanças no perfil das doenças e agravos à saúde ${ }^{7}$. A presença de uma ou mais doenças crônicas é significativamente maior entre as mulheres mais velhas, chegando a ser 62 vezes maior naquelas com idade $\geq 70$ anos em comparação às mulheres na faixa etária 30-39 anos ${ }^{8}$. Isto pode ocorrer devido à diminuição do envolvimento em atividades físicas moderadas e vigorosas, considerada esta como aquela que apresenta um custo energético médio entre 3,0 e 6,0 METs (taxa metabólica de repouso) e superior a 6,0 METs, respectivamente. Além disso, observa-se uma relação com o aumento progressivo da idade, que acarreta ao decréscimo da capacidade física. Sugere-se que a morbimortalidade entre homens e mulheres associada às doenças crônicas poderia ser reduzida com mudanças no estilo de vida, principalmente na dieta e na inclusão da atividade física ${ }^{7-9}$.

Dados provenientes de uma pesquisa realizada pelo Ministério da Saúde em capitais brasileiras determinaram que os homens são mais ativos que as mulheres, devido à pratica de atividade física como lazer ${ }^{10}$. Especificamente na Região Sul tem se observado maior prevalência de atividade física, sobretudo no estado de Santa Catarina. Na cidade de Florianópolis, o percentual de indivíduos que praticam atividade física chegou a $41,4 \%$, considerada a cidade mais ativa do país, sendo que entre os homens foi de $53 \%$ e mulheres $30,9 \%$. A menor prevalência de atividade física entre as mulheres, quando comparadas aos homens, também foi observada em Belo Horizonte, Manaus, Rio Branco e Belém ${ }^{11}$.

É possível observar uma menor tendência das mulheres à prática de atividade física ${ }^{12-14}$, podendo ser resultado dos padrões culturais ainda existentes na sociedade, pois além de exercerem uma atividade profissional, são por vezes as principais responsáveis pelos cuidados com o lar, marido e filhos ${ }^{15,16}$. Em uma população de adultos em Minas Gerais, 0 percentual de indivíduos que atingiu os 150 minutos foi cerca de três vezes maior entre os homens em comparação às mulheres no lazer e nos deslocamentos ${ }^{17}$. No entanto, as atividades relacionadas ao trabalho doméstico são relevantes no total de atividades desempenhadas pelas mulheres, pois de maneira geral, são representativas na rotina diária. Em alguns casos, as mulheres envolvidas nessas práticas apresentam níveis mais elevados de atividade física, quando comparadas àquelas que não realizam trabalho doméstico ${ }^{18,19}$.

A literatura tem demonstrado estudos voltados à população adulta de ambos os sexos na região do estado de Santa Catarina, nomeadamente nas cidades de Lages e Joaçaba, sendo encontrado alto nível de inatividade física na cidade de Joaçaba, com 57,4\% (IC95\%:53,4-61,4) e baixos níveis de inatividade física na cidade de Lages, com 29,6\% (IC95\%:27,6$31,7)$. Entretanto não há uma comparação entre os níveis de atividade física com outras regiões do estado ${ }^{19,20}$. Desta forma, percebe-se uma lacuna na literatura no intuito de identificar o nível de atividade física de mulheres de diferentes regiões do estado de Santa Catarina, principalmente nas regiões Serrana e Norte, uma vez que trata-se de regiões tipicamente diferentes em relação ao clima, economia, lazer e profissão. Nesse sentido, o presente estudo comparou a atividade física habitual das mulheres da região Norte e Serrana de Santa Catarina.

\section{Metodologia}

Este estudo de corte transversal faz parte de uma pesquisa mais ampla intitulada "Prevalência de atividade física de mulheres de Santa Catarina", aprovada pelo Comitê de Ética e Pesquisa em Seres Humanos da Universidade do Estado de Santa Catarina - protocolo $n^{0} 214 / 2011$. A amostra foi composta por uma população de aproximadamente 203.264 mulheres de 20 a 59 anos de acordo com o último censo do Instituto Brasileiro de Geografia e Estatística (2010), residentes das cidades de Joinville e Lages, por meio do critério de inclusão do estudo, ambas localizadas em Santa 
Catarina. Como critério de inclusão a cidade participante deveria ter mais de 150 mil habitantes de acordo com o Censo 201021, sendo uma cidade da região norte e outra da região Serrana. Apesar de ser uma amostra não probabilística, foi realizado o cálculo do tamanho da amostra para cada cidade segundo Barbetta22. 0 cálculo foi baseado na população de mulheres de 20 a 59 anos da cidade de Joinville - SC (157.177) e mulheres da cidade de Lages-SC (46.087)21. 0 cálculo da amostra mostrou a necessidade de um total de 396 mulheres para cada cidade com erro de estimativa de $5 \%$ e intervalo de confiança de $95 \%$. Ao final da coleta obteve-se uma amostra total de 705 mulheres sendo 352 mulheres de Joinville e 353 mulheres de Lages.

As participantes foram convidadas a participar do estudo voluntariamente, por meio de instituições empregadoras ou prestadoras de serviços no nível da educação, saúde, justiça, religião e seguros, além de participantes como donas de casa. Essas instituições tinham caráter comercial, como lojas de eletrodomésticos, vestuário, farmácias, panificadoras, supermercados, entre outras. Além disso, igrejas, escolas e universidades públicas e particulares também foram convidadas a participar da pesquisa. Foram incluídas neste estudo mulheres que apresentassem idade entre 20 a 59 anos residentes em Lages ou Joinville. Excluídas mulheres que apresentaram alguma doença clinicamente diagnosticada, como câncer e paraplegias, bem como mulheres institucionalizadas (asilos, presídios, hospitais) e aquelas que não tiveram capacidade de responder e/ou compreender o questionário.

A fim de se abranger um maior número de mulheres na faixa etária de 20 a 59 anos, a coleta de dados ocorreu de duas formas, por e-mail (encaminhado às participantes do estudo) num total de 174 questionários preenchidos e presencial com a presença da pesquisadora in loco (nas ruas, repartições, lojas, shopping center, centros comerciais, praias, igrejas...), com 531 questionários preenchidos, perfazendo um total de 705 questionários. Foram distribuídos 1260 questionários (700 presenciais e 560 online), sendo que apenas 705 foram devolvidos corretamente preenchidos. Em relação aos demais questionários, é importante ressaltar que 55 questionários impressos estavam preenchidos de maneira incorreta, 30 voltaram em branco e 84 foram perdidos nos locais da pesquisa. Quanto aos questionários enviados online, 73 deles foram imperfeitamente respondidos e 313 não retornaram.

Para a coleta dos dados foi utilizado questionário autoaplicável, composto de instrumentos validados, dividido em três partes:

a) Identificação pessoal: inquiriu sobre idade, escolaridade, estado civil, número de filhos, cor da pele, massa corporal e estatura (autoreferida) para cálculo do Índice de Massa Corporal - IMC (peso/estatura2). Para classificação do IMC (status de peso) se utilizou o protocolo da Organização Mundial de Saúde ${ }^{23}$ que estabelece os pontos de corte: abaixo do peso (IMC<18,5); peso normal (IMC 18,5-24,9); sobrepeso (IMC 25,0-29,9) e obesidade (IMC>30,0). Para fins estatísticos as categorias foram reagrupadas resultando em: Peso normal: abaixo do peso + peso normal; Acima do peso: sobrepeso + obesidade.

b) O estrato econômico foi avaliado por meio do critério padrão de classificação econômica Brasil 2010 - Associação Brasileira de Empresas de Pesquisa (ABEP), considerado o principal instrumento de segmentação da população segundo seu poder de compra, o qual classifica a população em classes econômicas $A, B, C, D$ e $E$, por meio da conversão da soma de pontos referentes ao grau de instrução da mulher pesquisada e a quantidade de bens de consumo existentes em casa24.

c) Nível de atividade física: avaliado por meio do Questionário Internacional de Atividade Física - International Physical Activity Questionnaire (IPAQ - versão curta). No Brasil, este questionário foi validado pelo Centro de Estudos do Laboratório de Aptidão Física de São Caetano do Sul - CELAFISCS, que é o centro coordenador do IPAQ no Brasil|25. Na avaliação da atividade física habitual por meio do IPAQ, quantificou-se o número de vezes em que um indivíduo realizou ao menos 10 minutos contínuos de caminhada, de atividade física de intensidade moderada e de intensidade vigorosa na última semana, em diversos envolvimentos, nomeadamente, laboral, doméstico, lazer, recreativo e desportivo. A atividade física foi classificada em caminhada, atividade física moderada, moderada + vigorosa, vigorosa e total em minutos/dia. O IPAQ classifica as participantes como sedentárias, insuficientemente ativas, ativas ou muito ativas a partir da verificação do número de vezes que as mesmas realizaram atividade física por mais de 10 minutos contínuos nos sete dias anteriores ao preenchimento do questionário.

A análise estatística foi realizada por meio do Statistical Package for Social Sciences (SPSS) - versão 20.0. Fez-se uso da estatística descritiva, mediante o cálculo de média, desvio padrão e percentual. Para a comparação das variáveis da atividade física (caminhada, atividade física moderada e vigorosa) com cada região (Norte e Serrana) se utilizou a ANOVA one-way. A prevalência dos níveis de atividade física (insuficientemente ativo, ativo e muito ativo) foi expressa em percentagem, determinada a partir da razão entre o número de participantes em cada nível de atividade física e 0 
número total de participantes em região. A comparação dos níveis de atividade física entre as duas regiões e os fatores demográficos foi efetuada com o teste do Qui quadrado e o V de Cramer, a fim de verificar a associação entre o nível de atividade física habitual com os fatores demográficos e status do peso. 0 nível de significância estatístico adotado nas análises de comparação entre grupos e de associações entre variáveis foi de $p<0,05$.

\section{Resultados}

Na tabela 1 são apresentadas as características das participantes do estudo divididas entre as cidades pesquisadas, nomeadamente o grupo 1 (Lages) e grupo 2 (Joinville). Observou-se que a maioria das mulheres é branca (83,7\%), possui ensino médio completo (44,4\%), pertence a classe econômica B (47,3\%), é jovem $(59,7 \%)$ e não tem filhos ( $54 \%$ ).

Tabela 1- Características das participantes do estudo de acordo com a região estudada. Lages e Joinville - SC. 2013

\begin{tabular}{|c|c|c|c|c|}
\hline & Total & $\begin{array}{l}\text { Grupo } 1 \\
\text { (Lages) }\end{array}$ & $\begin{array}{c}\text { Grupo } 2 \\
\text { (Joinville) }\end{array}$ & Valor de $p$ \\
\hline \multicolumn{5}{|l|}{ Cor da pele (\%) } \\
\hline Branca & 83,7 & 83,8 & 83,5 & \multirow{3}{*}{0,002} \\
\hline Negra & 6,4 & 3,7 & 9,1 & \\
\hline Parda & 9,9 & 12,5 & 7,4 & \\
\hline \multicolumn{5}{|l|}{ Escolaridade (\%) } \\
\hline Ensino Fundamental & 12,3 & 10,2 & 14,5 & \multirow{3}{*}{0,002} \\
\hline Ensino Médio & 44,4 & 51,0 & 37,8 & \\
\hline Ensino Superior & 43,3 & 38,8 & 47,7 & \\
\hline \multicolumn{5}{|c|}{ Estado conjugal (\%) } \\
\hline Sem companheiro & 47,6 & 45,5 & 49,7 & \multirow[b]{2}{*}{0,268} \\
\hline Com companheiro & 52,4 & 54,5 & 50,3 & \\
\hline \multicolumn{5}{|c|}{ Status do peso (\%) } \\
\hline Peso normal & 68,4 & 68,3 & 68,5 & \multirow[b]{2}{*}{0,966} \\
\hline Acima do peso & 31,6 & 31,7 & 31,5 & \\
\hline \multicolumn{5}{|c|}{ Estrato Econômico (\%) } \\
\hline $\mathrm{A}$ & 11,0 & 1,2 & 21,0 & \multirow{4}{*}{$<0,001$} \\
\hline$B$ & 47,3 & 39,5 & 55,1 & \\
\hline C & 28,3 & 49,1 & 7,4 & \\
\hline $\mathrm{D}$ & 13,4 & 10,2 & 16,5 & \\
\hline \multicolumn{5}{|l|}{ Faixa etária (\%) } \\
\hline 20-32 anos & 59,7 & 68,5 & 51,4 & \multirow{3}{*}{$<0,001$} \\
\hline $33-45$ anos & 21,5 & 19,5 & 23,3 & \\
\hline $46-59$ anos & 18,8 & 12,0 & 25,3 & \\
\hline \multicolumn{5}{|l|}{ Filhos (\%) } \\
\hline Nenhum & 54,0 & 57,5 & 50,6 & \multirow{3}{*}{$<0,001$} \\
\hline 1 a 2 filhos & 35,9 & 36,8 & 34,9 & \\
\hline 3 a 4 filhos & 10,1 & 5,7 & 14,5 & \\
\hline
\end{tabular}

Teste qui-quadrado $p<0,05$.

A prática da atividade física habitual é apresentada na tabela 2. Foi verificada diferença significativa na caminhada $(p<0,001)$ e na atividade física total $(p<0,001)$, nas quais observou-se que as mulheres de Lages apresentaram maior quantidade de tempo dedicado a estas variáveis $(55 \pm 51 \mathrm{~min} / \mathrm{d})$ e $(156 \pm 125)$, respectivamente, quando comparadas às mulheres de Joinville $(36 \pm 31 \mathrm{~min} / \mathrm{d})$ e $(126 \pm 88)$, respectivamente. Em relação ao tempo em que permanecem sentadas durante a semana e finais de semana, as mulheres de Joinville apresentaram as maiores médias $(p<0,001$; $p=0,001$ ) respectivamente. 
Tabela 2 - Prática da atividade física habitual das mulheres de acordo com a região. Lages e Joinville - SC. 2013.

\begin{tabular}{c|c|c|c}
\hline & Grupo 1 (Lajes) & Grupo 2 (Joinville) & Valor de p \\
\hline Caminhada (min/d) & $55 \pm 51$ & $36 \pm 31$ & $<\mathbf{0 , 0 0 1}$ \\
\hline AF mod. (min/d) & $61 \pm 63$ & $54 \pm 49$ & 0,074 \\
\hline AF vig. (min/d) & $40 \pm 56$ & $36 \pm 42$ & 0,256 \\
\hline AF mod+vig (min/d) & $101 \pm 99$ & $90 \pm 75$ & 0,090 \\
\hline AF total $(\mathrm{min} / \mathrm{d})$ & $156 \pm 125$ & $126 \pm 88$ & $<\mathbf{0 , 0 0 1}$ \\
\hline Sent./semana $(\mathrm{min} / \mathrm{d})$ & $1830 \pm 1114$ & $2490 \pm 1688$ & $<\mathbf{0 , 0 0 1}$ \\
\hline Sent./fds. $(\mathrm{min} / \mathrm{d})$ & $783 \pm 544$ & $880 \pm 456$ & $\mathbf{0 , 0 1 1}$ \\
\hline
\end{tabular}

AF. Atividade física; mod. Moderada; vig. Vigorosa; min/d; minuto/dia; Sentado/sem. Sentado semana; Sentado/fds. Sentado final de semana. ANOVA - $p<0,05$

Quanto ao nível de atividade física habitual das participantes do estudo (tabela 3), é possível observar que 71,7\% das mulheres são classificadas como suficientemente ativas (ativas + muito ativas), com destaque para as mulheres classificadas como ativas em Joinville $(39,2 \%)$ e em Lages como muito ativas $(45 \%) \quad(p<0,001)$.

Tabela 3 - Nível de atividade física habitual das mulheres de acordo com região. Lages e Joinville - SC. 2013.

\begin{tabular}{c|c|c|c|c}
\hline & Total & Grupo 1 (Lajes) & $\begin{array}{c}\text { Grupo 2 } \\
\text { (Joinville) }\end{array}$ & Valor de p \\
\hline $\begin{array}{c}\text { Insuficientemente } \\
\text { Ativa (\%) }\end{array}$ & 28,4 & 27,0 & 27,3 & $<\mathbf{0 , 0 0 1}$ \\
\hline Ativa (\%) & 32,8 & 28,0 & 39,2 & \\
\hline Muito ativa (\%) & 38,9 & 45,0 & 33,5 & \\
\hline
\end{tabular}

Nível de AF. Nível de atividade física habitual. Teste qui- quadrado $p<0,05$.

A associação dos fatores demográficos de acordo com o nível de atividade física das participantes do estudo é apresentada na tabela 4, na qual observou-se, apesar de fraca associação em ambas as variáveis $(v=0,184 ; \mathrm{v}=0,175)$, que as mulheres que possuem ensino médio mostraram-se muito ativas $(48 \%)(p=0,001)$ e aquelas que pertencem ao estrato econômico B revelaram-se insuficientemente ativas (53\%). 
Tabela 4 - Comparação e associação do nível de atividade física habitual com os fatores demográficos e status do peso em mulheres. Lages e Joinville - SC. 2013.

\begin{tabular}{|c|c|c|c|c|c|c|}
\hline & Total & $\begin{array}{c}\text { Insuficientemente } \\
\text { Ativa }\end{array}$ & Ativa & $\begin{array}{l}\text { Muito } \\
\text { Ativa }\end{array}$ & Valor de $p$ & $\begin{array}{c}\text { V de } \\
\text { Cramer }\end{array}$ \\
\hline \multicolumn{7}{|c|}{ Escolaridade (\%) } \\
\hline $\begin{array}{c}\text { Ensino } \\
\text { fundamental }\end{array}$ & 12,3 & 8,0 & 11,3 & 15,6 & \multirow{3}{*}{0,001} & \multirow{3}{*}{0,184} \\
\hline Ensino médio & 44,4 & 44,5 & 40,2 & 48,8 & & \\
\hline Ensino superior & 43,3 & 47,5 & 48,5 & 36,4 & & \\
\hline \multicolumn{7}{|c|}{ Estado conjugal (\%) } \\
\hline Morando junto & 47,6 & 49,0 & 45,0 & 48,7 & \multirow[b]{2}{*}{0,748} & \multirow{2}{*}{0,042} \\
\hline $\begin{array}{l}\text { Morando } \\
\text { separado }\end{array}$ & 52,4 & 51,0 & 55,0 & 51,3 & & \\
\hline \multicolumn{7}{|c|}{ Status de peso (\%) } \\
\hline Peso normal & 68,4 & 70,0 & 68,3 & 66,8 & \multirow{2}{*}{0,493} & \multirow{2}{*}{0,059} \\
\hline Acima do peso & 31,6 & 30,0 & 31,7 & 33,2 & & \\
\hline
\end{tabular}

Estrato econômico (\%)

\begin{tabular}{|c|c|c|c|c|c|c|}
\hline$A$ & 11,1 & 13,5 & 10,0 & 10,4 & \multirow{4}{*}{0,010} & \multirow{4}{*}{0,175} \\
\hline B & 47,2 & 53,0 & 48,9 & 41,8 & & \\
\hline $\mathrm{C}$ & 28,3 & 27,0 & 23,8 & 32,5 & & \\
\hline D & 13,4 & 6,5 & 17,3 & 15,3 & & \\
\hline \multicolumn{7}{|c|}{ Faixa etária (\%) } \\
\hline 20-32 anos & 59,7 & 67,4 & 54,0 & 58,6 & \multirow[t]{3}{*}{0,135} & \multirow[t]{3}{*}{0,119} \\
\hline $33-45$ anos & 21,5 & 18,6 & 23,5 & 21,8 & & \\
\hline 46-59 anos & 13,4 & 14,0 & 22,5 & 19,6 & & \\
\hline
\end{tabular}

Filhos (\%)

\begin{tabular}{c|c|c|c|c|c|c}
\hline Nenhum & 54,0 & 58,5 & 51,5 & 53,2 & \multirow{2}{*}{0,075} & 0,127 \\
\hline 1 a 2 filhos & 35,9 & 35,5 & 33,8 & 37,5 & & \\
\hline 3 a 4 filhos & 10,1 & 6,0 & 14,7 & 9,3 & & \\
\hline
\end{tabular}

Valor de p: Nível de significância relativo à comparação da amostra entre os grupos efetuada por meio do teste qui-quadrado ( $p<$ 0,05).

\section{Discussão}

Diante do objetivo do presente estudo que foi comparar a atividade física habitual das mulheres da região Norte e Serrana de Santa Catarina, os resultados indicaram que $71,7 \%$ das mulheres foram classificadas como suficientemente ativas (ativas + muito ativa) e as duas regiões estudadas apresentaram alta prevalência de atividade física, $73 \%$ para região Serrana e 72,7\% para região Norte. Apesar de utilizar o mesmo instrumento de medida (IPAQ), esses resultados diferem de resultados já encontrados em Santa Catarina, especificamente na cidade de Joaçaba, em que o IPAQ foi empregado na forma de entrevista, com uma prevalência de $42,6 \%$ de atividade física tanto de homens quanto de mulheres ${ }^{20}$. Essa maior prevalência de atividade física apresentada em nosso estudo, pode em parte ser atribuída ao fato de que os entrevistados apresentam dificuldade em distinguir as atividades moderadas e vigorosas e com isso, podem ter superestimado sua prática de atividade física ${ }^{26-28}$. Entretanto, cabe ressaltar, que o IPAQ é o instrumento mais utilizado em estudos brasileiros e internacionais sobre atividade físiาca, pela facilidade de aplicação em grandes amostras e por possibilitar a comparabilidade entre estudos ${ }^{29}$.

Ao comparar a prevalência de atividade física das mulheres das duas regiões neste estudo, pode-se observar que ambas as regiões obtiverem bons escores em relação ao nível de atividade física. Provavelmente isso esteja relacionado ao fato de que Lages e Joinville dispõem de academias nas praças públicas, contando com aparelhos e Profissionais de Educação Física que auxiliam e incentivam a prática de atividade física da população ${ }^{19}$. Estudos demonstram que 
um local adequado favorece a prática de atividade física ${ }^{19,36}$. No caso de Joinville, há em sua estrutura $115 \mathrm{~km}$ de vias cicláveis ${ }^{30}$, facilitando dessa forma, o uso da bicicleta e aumentando a prática de atividade física, uma vez que $12 \%$ dos deslocamentos em Joinville são feitos por bicicletas, muito acima da média nacional de 1,75\% ${ }^{31}$.

Quanto à intensidade da atividade física apresentada pelas mulheres deste estudo, pode-se observar que as mulheres de ambas as cidades dedicavam maior tempo de atividade física na intensidade moderada (61 $\pm 63 \mathrm{~min} / \mathrm{d}$ Lages; $54 \pm 49 \mathrm{~min} / \mathrm{d}$ - Joinville). No entanto, não houve diferença entre as regiões estudadas. A diferença significativa foi relatada para a caminhada e para a atividade física total $(<0,001 ;<0,001)$, respectivamente. As mulheres da região Serrana reportaram maior prática de caminhada $(55 \pm 51 \mathrm{~min} / \mathrm{d})$ quando comparadas as da região Norte $(38 \pm 31 \mathrm{~min} / \mathrm{d})$. Da mesma forma, Zanchetta et al. ${ }^{32}$ verificaram em seu estudo, que entre as modalidades praticadas no lazer, a caminhada foi a mais prevalente nas mulheres com 16,7\%. Outro estudo também verificou que a caminhada é mais frequentemente realizada pelas mulheres $(48 \pm 43 \mathrm{~min} / \mathrm{d})$, representando aproximadamente $70 \%$ da atividade física diária total ${ }^{33}$. Segundo Rech et al. ${ }^{34}$ a caminhada é uma atividade comum ao ser humano, enquanto as atividades físicas moderadas e vigorosas envolvem maior complexidade de ações motoras, além de maior intensidade. Atrelado a este contexto, a caminhada além de ser frequentemente utilizada como exercício físico, particularmente entre indivíduos com menor aptidão física, constitui uma forma ativa de deslocamento ${ }^{33}$. Em países do norte da Europa, como na Finlândia, Suécia e Irlanda o deslocamento ativo, por meio da caminhada ou da bicicleta, contribui de forma significativa para o cumprimento da recomendação da acumulação dos 30 minutos diários de atividade física ${ }^{35}$.

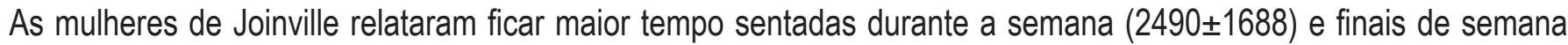

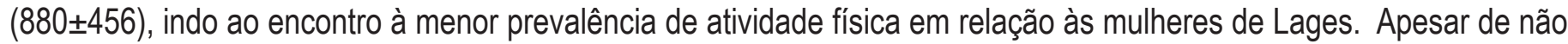
ter sido avaliado o momento profissional dessas mulheres, é possível elucidar, com base na faixa etária apresentada por elas (51,4\% pertencem a faixa etária de 20-32 anos), que as mesmas estão solidificando suas carreiras, dessa forma, passam um maior tempo sentadas estudando e/ou trabalhando, dispondo de menor tempo para a prática de atividade física ${ }^{36}$. Alguns estudos científicos afirmam que a falta de tempo e a jornada de trabalho ou de estudos são usualmente apontados como os principais empecilhos para adesão à prática de atividade física entre indivíduos de diferentes faixas etárias e categorias profissionais ${ }^{37-39}$. É importante destacar ainda, que o tempo gasto sentado está diretamente ligado ao aparecimento de doenças, especificamente ao aumento da obesidade, hipertensão e síndrome metabólica ${ }^{40}$.

Estudos apontam que a escolaridade, o número de filhos, o estado nutricional, o estado conjugal e o estrato socioeconômico são fatores decisivos para um estilo de vida ativo ${ }^{41-44,11}$. Contudo, no presente estudo, houve associação apenas com a escolaridade e o estrato econômico $(p=0,001 ; 0,010)$ respectivamente. Dessa forma, pode-se observar que as mulheres com ensino médio foram consideradas muito ativas, apesar da pequena diferença em relação às mulheres do ensino superior (12\%). Estes resultados discordam da literatura, pois segundo os estudos de MartinezGonzalez et al. ${ }^{45}$ Matsudo et al. ${ }^{43}$ e VIGITEL11 indivíduos com mais anos de escolaridade demonstram geralmente maior atividade física devido a diferenças na condição financeira, e consequentemente na oportunidade de acesso aos recursos e às orientações para a saúde. Em relação ao estrato socioeconômico, pode-se observar que as mulheres classificadas com estrato econômico B apresentaram-se mais insuficientemente ativas em relação às classificadas em outros estratos. Estes achados corroboram com os resultados encontrados em outros estudos, nos quais a inatividade física foi mais prevalente entre indivíduos de maior renda mensal ${ }^{18,20,46}$.

Como na maior parte dos estudos sobre atividade física, o presente estudo foi avaliado por meio de um questionário, o que pode representar uma limitação deste estudo. Apesar das restrições relacionadas ao instrumento, os resultados deste estudo poderão contribuir para o avanço científico nesta temática, além de permitir comparações e novas reflexões em outros contextos.

\section{Considerações Finais}

Os resultados evidenciam que as mulheres de ambas as regiões são ativas, no entanto, apresentando diferença mínima, as mulheres residentes em Lages foram consideradas mais ativas, realizando maior tempo de atividade física habitual, com destaque para prática da caminhada. Desta forma, pode-se concluir que as mulheres das duas regiões estudadas atendem a recomendação da Organização Mundial da Saúde (2011) para a prática de atividade física e acumulam pelo menos 30 minutos de atividade física moderada diariamente. 
Por fim, cabe ressaltar a necessidade de novas pesquisas que aprofundem os aspectos trabalhados neste estudo, estendendo-os a outras populações e contextos socioculturais, envolvendo maior número de participantes a fim de verificar a generalização destes dados.

\section{Referências Bibliográficas}

1. Chaput JP, Saunders TJ, Mathieu ME, Henderson M, Tremblay MS, O'Loughlin J et al. Combined associations between moderate to vigorous physical activity and sedentary behaviour with cardiometabolic risk factors in children. Appl. Physiol. Nutr. Metab. 2013; 38 (5):477-483.

2. Cipriani NCS, Meurer ST, Benedetti TRB, Lopes MA. Aptidão funcional de idosas praticantes de atividades físicas. Rev bras cineantropom desempenho hum. 2010; 12 (2): 106-111.

3. Guimarães AC A, Baptista, F. Influence of habitual physical activity on the symptoms of climacterium/menopause and the quality of life of middle-aged women. Int J Womens Health, 2011; 3: 319-328.

4. Ceschini FL, Andrade DR, Oliveira LC, Araújo Júnior JF, Matsudo VK. Prevalence of physical inactivity and associated factors among high school students from state's public schools. J Pediatr (Rio J). 2009; 85 (4):301-6

5. World Health Organization (US). Global Recommendations on Physical Activity for Health, 2010.

6. Haskell WL, Lee I-M, Pate RR, Powell KE, Blair SN, Franklin BA et al. Physical activity and public health: updated recommendation for adults from the American College of Sports Medicine and the American Heart Association. Med Sci Sports Exerc. Special reports. 2007; 116: 1423-1434.

7. Ferreira M, Matsudo S, Matsudo V, Braggion G. Efeitos de um programa de orientação de atividade física e nutricional sobre o nível de atividade física de mulheres fisicamente ativas de 50 a 72 anos de idade. Rev Bras Med Esporte. 2005; 11(3): 172-176.

8. Barreto SM, Figueiredo RC. Doença crônica, auto-avaliação de saúde e comportamento de risco: diferença de gênero. Rev Saúde Públ. 2009; 43(Suppl 2): 38-47.

9. Moretti, AC, Almeida V, Westphal MF, Bógus CM.. Práticas corporais/atividade física e políticas públicas de promoção da saúde. Saúde soc. 2009; 18(2): 346-354.

10. Ministério da saúde (Brasil). Estimativas sobre frequência e distribuição sócio demográfica de fatores de risco e proteção para doenças crônicas nas capitais dos 26 estados brasileiros e no Distrito Federal em 2011, 2012.

11. Vigitel Brasil 2011: Vigilância de Fatores de Risco e Proteção para Doenças Crônicas por Inquérito Telefônico. Ministério da Saúde, Secretaria de Vigilância em Saúde - Brasília: Ministério da Saúde, 2012.

12. Bauman A, Bull F, Chey T, Craig CL, Ainsworth BE, Sallis JF et al. The International Prevalence Study on Physical Activity: results from 20 different countries. Int J Behav Nutr Phys Act. 2009; 6 (89): 21-32.

13. Sávio KEO, Costa THM da, Schmitz BAS, Silva EF da. Sexo, renda e escolaridade associados ao nível de atividade física de trabalhadores. Rev Saúde Públ. 2008; 42(3): 457-463.

14. Silva RB, Costa-Paiva L, Pinto NAM, Braga AA, Morais SS. Atividade física habitual e risco cardiovascular na pósmenopausa. Rev Assoc Med Bras. 2006; 52 (4): 242-246.

15. Boran A, Shawaheen M, Khader Y, Amarin Z, Hill Rice V. Work-related stress among health professionals in northern Jordan. Occup Med. 2012; 62(2): 145-147.

16. Vieira JA. A identidade da mulher na modernidade. DELTA. 2005; 21 (spe): 207-238.

17. Bicalho PG, Hallal PC, Gazzinelli A, Knuth AG, Velásquez-Meléndez G. Atividade física e fatores associados em adultos de área rural em Minas Gerais, Brasil. Rev Saúde Públ. 2010; 44(5): 884-893.

18. Hallal PC, Victora CG, Wells JC, Lima RC. Physical inactivity: prevalence and

associated variables in Brazilian adults. Med Sci Sports Exerc. 2003; 35 (11): 1894-1900.

19. Lopes JA, Longo GZ, Peres KG, Boing AF, Arruda MP. Fatores associados à atividade física insuficiente em adultos: estudo de base populacional no sul do Brasil. Rev. bras epidemiol. 2010; 13 (4): 689-698.

20. Baretta E, Baretta M, Peres KG. Nível de atividade física e fatores associados em adultos no Município de Joaçaba, Santa Catarina, Brasil. Cad. Saúde Pública 2007; 23 (7): 1595-1602.

21. Instituto Brasileiro De Geografia E Estatística (Brasil). Projeção da população do Brasil/censo 2010. Disponível em: http//www.ibge.gov.br. Acesso em: 02 de maio de 2012. 
22. Barbetta PA. Estatística aplicada às ciências sociais. 7 ed. rev. Florianópolis: Editora da UFSC, 2008.

23. World Health Organization (US). Global Strategy on Diet, Physical Activity and Health, 2004.

24. Associação Brasileira De Empresas De Pesquisa (Brasil). Critério de Classificação Econômica Brasil, 2010.

25. Pardini R, Matsudo S, Matsudo TAV, Andrade E, Braggion G, Andrade D et al. Validation of the international physical activity questionnaire (IPAQ): pilot study in brazilian young adults. Med Sci Sports Exerc. 1997; 29: S5-S9.

26. Ainsworth BE, Macera CA, Jones DA, Reis JP, Addy CL, Bowles HR et al. Comparison of the 2001 BRFSS and the IPAQ physical activity questionnaires. Med Sci Sports Exerc. 2006; 38(9): 1584-1592.

27. Rzewnicki R, Auweele YV, Bourdeaudhuij I. Addressing overreporting on the International Physical Activity Questionnaire (IPAQ) telephone survey with a population sample. Public Health Nutr. 2003; 6(3): 299-305.

28. Sallis JF, Saelens BE. Assessment of physical activity by self-report: status, limitations, and future directions. Res $Q$ Exerc Sport. 2000; 71(2Suppl):S1-S14.

29. Hallal PC, Dumith SC, Bastos JP, Reichert FF, Siqueira FV, Azevedo MR. Evolução da pesquisa epidemiológica em atividade física no Brasil: revisão sistemática. Rev Saúde Pública. 2007;41( 3 ): 453-460.

30. Prefeitura Municipal de Joinville (Brasil). Joinville Cidade em dados 2013. Disponível em: http://www.joinville.sc.gov. br/. Data de acesso: 22 de setembro de 2013.

31. Prefeitura Municipal de Joinville (Brasil). Pesquisa Origem/Destino. Pesquisa Domiciliar Parte III- Características dos Deslocamentos. v. 2, 2010. Disponível em: http://www.joinville.sc.gov.br/. Data de acesso: 22 de setembro de 2013.

32. Zanchetta LM, Barros MBA, César CLG, Carandina L, Goldbaum M, Alves MCGP. Inatividade física e fatores associados em adultos, São Paulo, Brasil. Rev. bras. epidemiol. 2010; 13 (3): 387-399.

33. Guimaraes ACA, Baptista F. Prevalence of sufficient physical activity in middle-aged women from a Brazilian state capital. Rev bras cineantropom desempenho hum. 2013; 5(6): 677-685.

34. Rech CR, Fermino RC, Hallal PC, Reis RS. Validade e fidedignidade da escala de satisfação com a prática de atividade física em adultos. Rev Saúde Públ. 2011; 45(2): 286-293.

35. Sjostrom M, Oja P, Hagströmer M, Smith BJ, Bauman A. Health-enhancing physical activity across European Union countries: the Eurobarometer study. J Public Health. 2006; 14(5): 291-300.

36. Sousa TFD, Fonseca AS, Barbosa AR. Perceived barriers by university students in relation the leisure-time physical activity. Rev Bras Cineantropom Desempenho Humano. 2013; 15(2):164-173.

37. Castro-Carvajal JA, Patiño-Villada FA, Cardona-Rendón BM, Ochoa- Patiño V. Aspectos Asociados a la Actividad Física en el Tiempo Libre en la Población Adulta de un Municipio Antioqueño. Rev Salud Pública. 2008; 10(5): 679-690. 38. Dunton GF, Schneider M. Perceived barriers to walking for physical activity. Prev Chronic Dis. 2006; 3(4): 1-11.

39. Reichert FF, Barros AJ, Domingues MR, Hallal PC. The role of perceived personal barriers to engagement in leisuretime physical activity. Am J Public Health. 2007; 97(3): 515-9.

40. Owen N, Healy GN, Matthews CE, Dunstan DW. Too much sitting: the population health science of sedentary behavior. Exerc Sport Sci Rev. 2010; 38(3):105-113.

41. Camões M, Lopes C. Fatores associados à atividade física na população portuguesa. Rev Saúde Públ. 2008; 42(2): 208-216.

42. Malta DC, Castro AM, Gosch CS, Cruz DKA, Bressan, Nogueira JD et al. A Política Nacional de Promoção da Saúde e a agenda da atividade física no contexto do SUS. Epidemiol Serv Saúde. 2009; 18(1): 79-86.

43. Matsudo SM, Matsudo VR, Araújo T, Andrade D, Andrade E, Oliveira L et al. Nível de atividade física da população do estado de São Paulo: análise de acordo com o gênero, idade, nível sócio econômico, distribuição geográfica e de conhecimento. RBCM. 2002; 10(4): 41-50.

44. Siqueira F V, Facchini LA, Piccini RX, Tomasi E, Thumé E, Silveira DS et al . Atividade física em adultos e idosos residentes em áreas de abrangência de unidades básicas de saúde de municípios das regiões Sul e Nordeste do Brasil. Cad. Saúde Pública. 2008; 24(1): 39-54.

45. Martínez-González MA, Varo JJ, Santos JL, Irala J de, Gibney M, Kearney J et al. Prevalence of physical activity during leisure time in the European Union. Med Sci Sports Exerc. 2001; 33(7):1142-1146.

46. Masson CR, Dias-da-Costa JS, Olinto MTA, Meneghel S, Costa CC da, Bairros F et al. Prevalência de sedentarismo nas mulheres adultas da cidade de São Leopoldo, Rio Grande do Sul, Brasil. Cad Saúde Pública. 2005; 21(6):1685-1694. 


\section{Camila da Cruz Ramos de Araujo}

Endereço para correspondência - Evaldo Schaeffer, 422, bloco B10 Apto 23. Bairro Jardim Atlântico, CEP 88095-350, Florianópolis, SC, Brasil.

E-mail: cami.ramosdearaujo@hotmail.com

Lattes: http://lattes.cnpq.br/0696770907183182

Alexandrina Gomes de Oliveira - alexandrina2312@gmail.com

Adriana Coutinho de Azevedo Guimarães - nanaguim@terra.com.br

Leonessa Boing - leonessaboing@gmail.com

Melissa de Carvalho Souza - mecarvalho.s@gmail.com

Zenite Machado-zenite13@yahoo.com.br

Enviado em 11 de fevereiro de 2014.

Publicado em 15 de setembro de 2014. 